\title{
Translational evaluation of novel selective orexin-1 receptor antagonist JNJ-61393215 in an experimental model for panic in rodents and humans
}

Giacomo Salvadore ${ }^{1}$, Pascal Bonaventure $\mathbb{D}^{2}$, Anantha Shekhar ${ }^{3}$, Philip L. Johnson ${ }^{4}$, Brian Lord², Brock T. Shireman², Terry P. Lebold ${ }^{2}$, Diane Nepomuceno ${ }^{2}$, Christine Dugovic ${ }^{2}$, Sander Brooks ${ }^{5,6}$, Rob Zuiker ${ }^{5}$, Cathy Bleys ${ }^{7}$, Kanaka Tatikola ${ }^{8}$, Bart Remmerie”, Gabriel E. Jacobs ${ }^{5,9}$, Koen Schruers ${ }^{10}$, John Moyer ${ }^{1}$, Abigail Nash', Luc G. M. Van Nueten ${ }^{7}$ and Wayne C. Drevets ${ }^{2}$

\begin{abstract}
Orexin neurons originating in the perifornical and lateral hypothalamic area project to anxiety- and panic-associated neural circuitry, and are highly reactive to anxiogenic stimuli. Preclinical evidence suggests that the orexin system, and particularly the orexin-1 receptor (OX1R), may be involved in the pathophysiology of panic and anxiety. Selective OX1R antagonists thus may constitute a potential new treatment strategy for panic- and anxiety-related disorders. Here, we characterized a novel selective OX1R antagonist, JNJ-61393215, and determined its affinity and potency for human and rat OX1R in vitro. We also evaluated the safety, pharmacokinetic, and pharmacodynamic properties of JNJ-61393215 in first-in-human single- and multiple-ascending dose studies conducted. Finally, the potential anxiolytic effects of JNJ61393215 were evaluated both in rats and in healthy men using 35\% $\mathrm{CO}_{2}$ inhalation challenge to induce panic symptoms. In the rat $\mathrm{CO}_{2}$ model of panic anxiety, JNJ-61393215 demonstrated dose-dependent attenuation of $\mathrm{CO}_{2^{-}}$ induced panic-like behavior without altering baseline locomotor or autonomic activity, and had minimal effect on spontaneous sleep. In phase-1 human studies, JNJ-61393215 at $90 \mathrm{mg}$ demonstrated significant reduction $(P<0.02)$ in $\mathrm{CO}_{2}$-induced fear and anxiety symptoms that were comparable to those obtained using alprazolam. The most frequently reported adverse events were somnolence and headache, and all events were mild in severity. These results support the safety, tolerability, and anxiolytic effects of JNJ-61393215, and validate $\mathrm{CO}_{2}$ exposure as a translational cross-species experimental model to evaluate the therapeutic potential of novel anxiolytic drugs.
\end{abstract}

\section{Introduction}

Orexin neuropeptides, also known as hypocretins, are critically involved in coordinating adaptive physiological, behavioral, and endocrine responses to salient stimuli or

\footnotetext{
Correspondence: Pascal Bonaventure (pbonave1@its.jnj.com)

'Janssen Research \& Development, LLC, Titusville, NJ, USA

${ }^{2}$ Janssen Research \& Development, LLC, San Diego, CA, USA

Full list of author information is available at the end of the article

Presented at: SOBP Annual Meeting,16-18 May 2019, Chicago, IL; ASCP Annual Meeting, 27-31 May 2019, Scottsdale, AZ.

These authors contributed equally: Giacomo Salvadore, Pascal Bonaventure
}

conditions, including stress and exposure to aversive or threatening stimuli. The orexin cell bodies are restricted to the tuberal hypothalamus, which plays key roles in regulating multiple physiological functions ${ }^{1,2}$. In addition to projections to other hypothalamic and brainstem nuclei, and to cortical and subcortical structures associated with medial prefrontal cortical network, the extensive distribution of axonal projections from orexin neurons to the central nucleus of the amygdala and the bed nucleus of the stria terminalis putatively supports the synchronization and

\section{(c) The Author(s) 2020}

(c) (i) Open Access This article is licensed under a Creative Commons Attribution 4.0 International License, which permits use, sharing, adaptation, distribution and reproduction cc) in any medium or format, as long as you give appropriate credit to the original author(s) and the source, provide a link to the Creative Commons license, and indicate if changes were made. The images or other third party material in this article are included in the article's Creative Commons license, unless indicated otherwise in a credit line to the material. If material is not included in the article's Creative Commons license and your intended use is not permitted by statutory regulation or exceeds the permitted use, you will need to obtain permission directly from the copyright holder. To view a copy of this license, visit http://creativecommons.org/licenses/by/4.0/. 
amplification of their firing activity in anxiety, fear, and motivated states ${ }^{3}$.

At the terminals of these projections, orexin interacts with two distinct receptors, orexin-1 (OX1R) and orexin-2 receptor (OX2R) subtypes. Preclinical studies have provided insights into the mechanism of orexin system as a wakefulness stabilizer in normal sleep-wake cycle ${ }^{4}$, and have shown that antagonism of OX2R is sufficient to induce and prolong sleep in rodents ${ }^{5}$ and humans ${ }^{6}$. The key role of the orexin system in sleep/wakefulness regulation is characterized by the specific anatomical distribution of OX2Rs in histaminergic neurons in the tuberomammillary nucleus ${ }^{7}$. In contrast, OX1Rs are more selectively expressed in bed nucleus of the stria terminalis, amygdala, cingulate cortex, and the noradrenergic neurons of the locus coeruleus ${ }^{8}$. Consistent with the anatomical distribution of OX1R, a critical role for this receptor is emerging in complex emotional behavior, such as association of OX1R pathway overactivation with panic or anxiety states ${ }^{9-13}$.

Orexin neurons originating in the perifornical and lateral hypothalamic area are highly reactive to anxiogenic stimuli, and have strong projections to anxiety- and panic-associated circuitry expressing $O X 1 R^{13,14}$. In rats, after administration of the anxiogenic inverse benzodiazepine agonist FG-7142 ${ }^{15}$, a selective OX1R antagonist blocked cellular responses in panic and anxiety brain circuits, and imaging data indicated that a selective OX1R antagonist produced a regiondependent inhibition of yohimbine-induced activation in fronto-hippocampal regions as well as in several key components of the extended amygdala ${ }^{7}$. The OX1R inhibition in amygdala is consistent with preclinical studies demonstrating that the orexin system is implicated in amygdala regulation of fear-associated learning ${ }^{8-10}$. In contrast, OX1R antagonists have minimal effect on sleep stages in rodents ${ }^{11,12}$. Overall, there is compelling evidence that overactivation of the OX1R pathway is associated with hyperexcited or hyperactive states; thus, conceptually, a selective OX1R antagonist might normalize overexcited networks without inducing sedation ${ }^{12}$. The extant data also indicated a link between hyperactive orexin system and anxiety- and panic vulnerability in rats and humans ${ }^{13}$. Activation of OX1R was also shown to be a critical component of $\mathrm{CO}_{2}$-mediated anxiety and hypertension ${ }^{16}$; notably, $\mathrm{CO}_{2}$ inhalation can induce panic-like symptoms in rodents and humans ${ }^{17}$.

JNJ-61393215, a selective OX1R antagonist, is under development for treatment of neuropsychiatric disorders, including stress- and fear-related anxiety syndromes arising within the context of mood and anxiety disorders. Here, we describe the pharmacologic characterization of JNJ61393215 , evaluating in vitro affinity and potency for human and rat OX1R by radioligand-binding and in vitro functional assays. In rats, we measured in vivo target engagement of JNJ-61393215 in the brain and its pharmacodynamic effects on sleep/wake cycles. In our previous studies, we have shown that while selective OX1R antagonism did not affect sleep-wake states, blockade of both OX1R and OX2R elicited a disinhibition of rapid eye movement (REM) sleep at the expense of non-REM (NREM) sleep in rodents ${ }^{11,12,18}$. Therefore, the effect of JNJ-61393215 on sleep was tested in OX2R-knockout (KO) mice to demonstrate in vivo functional target engagement. The activity of JNJ-61393215 was then evaluated on panicrelated behaviors induced using the $\mathrm{CO}_{2}$ inhalation model.

In addition, we report the first-in-human single-ascending dose $(\mathrm{SAD})$ and multiple-ascending dose (MAD) study wherein the safety, tolerability, pharmacokinetics (PK), and pharmacodynamics (PD) of JNJ-61393215 were evaluated in healthy participants. As inhalation of $35 \% \mathrm{CO}_{2}$ is a validated model for panic-like symptoms that is also sensitive to the panicolytic effects of benzodiazepines ${ }^{17,19,20}$, we also studied the potential anxiolytic effects of JNJ-61393215, using alprazolam as an active control, in healthy human participants subjected to fear-related anxiogenic effects of $\mathrm{CO}_{2}$ inhalation who showed sensitivity to the panic-inducing effects of this challenge during screening.

\section{Materials and methods \\ Animal experiments}

All animal experiments were conducted according to the standards recommended by the Guide for the Care and Use of Laboratory Animals adopted by the US National Institutes of Health (NIH Publication no. 80-23, revised 1996), and the Janssen Research \& Development, LLC, Animal Care and Use Committee approved the protocols. Animals were housed under controlled conditions with a 12-/12-h light/dark schedule and temperature of $22 \pm 2{ }^{\circ} \mathrm{C}$. Food and water were provided ad libitum. Experiments were performed after animals had acclimatized for at least 1 week unless stated otherwise.

\section{Test article}

OX1R antagonist JNJ-61393215 (chemical name: 3-fluoro2-(pyrimidin-2-yl)phenyl)((1 S,4 R,6 R)-6-((5-(trifluoromethyl)pyridin-2-yl)oxy)-2-azabicyclo[2.2.1] heptan-2$\mathrm{yl}-3,3-\mathrm{d} 2)$ methanone) and its less active enantiomer JNJ63821238 were synthetized at Janssen Research \& Development, LLC (Fig. 1A). Preparation of dosing formulations and reference standards is shown in supplementary text.

\section{In vitro radioligand-binding assays and in vitro functional assays}

These assays were performed as previously described ${ }^{11}$.

\section{Ex vivo receptor-occupancy assays and plasma/brain exposure measurements}

Male Sprague-Dawley rats (300-400 g, Charles River Laboratories, San Diego, CA) received a bolus dose of JNJ61393215 via a 16-gauge intragastric gavage of 10,1 , or 

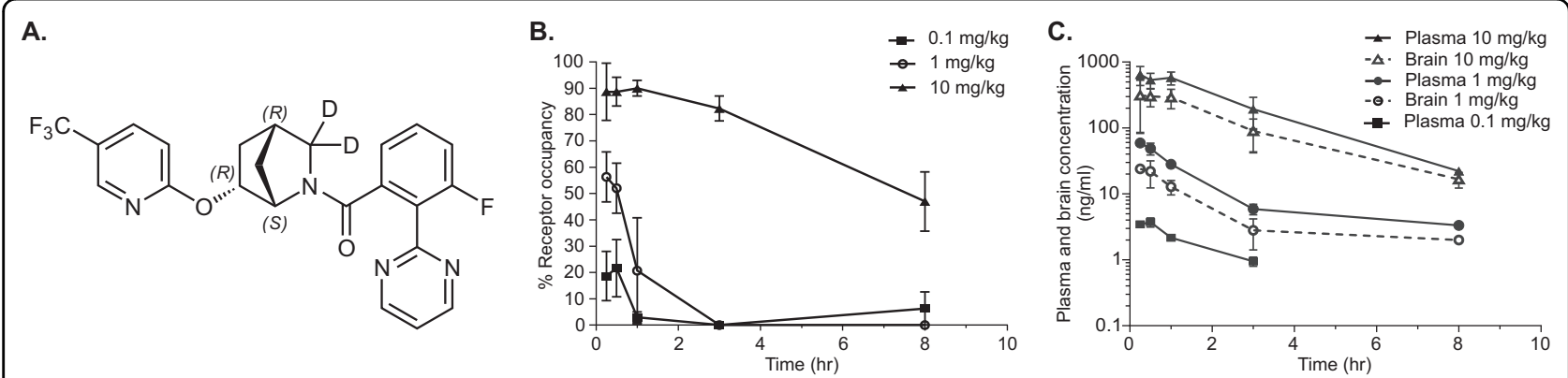

Fig. 1 A Chemical structure of 3-fluoro-2-(pyrimidin-2-yl)phenyl)((1 S,4 R,6 R)-6-((5-(trifluoromethyl)pyridin-2-yl)oxy)-2-azabicyclo[2.2.1]heptan-2-yl-3,3d2)methanone). B Ex vivo OX1R occupancy with JNJ-61393215 (10, 1, or $0.1 \mathrm{mg} / \mathrm{kg}$, oral administration) in rat tenia tecta: time dependency.

C Corresponding plasma and brain concentrations ( $n=3$ per time point and dose regimen, brain concentration at 0.1 mg/kg was not measured).

$0.1 \mathrm{mg} / \mathrm{kg}$. Rats were euthanized using $\mathrm{CO}_{2}$ and decapitated at defined time points $(0.2,0.5,1,3$, and $8 \mathrm{~h})$ after administration of test compound $(n=3$ per time point and dose regimen). Brain and plasma samples were processed as described in supplementary text. Receptor occupancy was measured in the tenia tecta due to the high OX1R-binding density observed in that region, as reported previously ${ }^{12}$.

\section{Sleep recording and analysis in rats and mice}

Sleep experiments were conducted in male Sprague-Dawley rats (350-450 g, Harlan Laboratories, Livermore, CA, USA) and in male C57B16 OX2R KO and corresponding wild-type (WT) mice (30-35 g, Charles River Laboratories, San Diego, CA). Sleep was assessed using implanted telemetry to record electroencephalographic (EEG) and electromyographic (EMG) signals using a crossover design as described previously ${ }^{21}$ and in supplementary text. To determine significant differences between vehicle and compound treatments, a paired Student's $t$ test was performed for NREM and REM latencies and total NREM and REM sleep durations for $6 \mathrm{~h}$. A two-way repeated measure (interaction time $\times$ treatment) analysis of variance (ANOVA) followed by a Bonferroni post hoc test was used for NREM and REM sleep duration per 2-h intervals. Differences were determined to be significant if $P<0.05$. No randomization was used, and blinding was not done.

\section{$20 \% \mathrm{CO}_{2}$-induced panic provocation model and cardiovascular response in rats}

Experiments were performed in male Sprague-Dawley rats (300-350 g, Harlan Laboratory, Indianapolis, IN) as previously described, using a counterbalanced design ${ }^{12}$. JNJ63821238, the less active enantiomer of JNJ-61393215, was included as a negative control, and was formulated in the same vehicle. The social interaction test, a fully validated test of experimental anxiety-like behavior in rats, was performed in the open-field arena ${ }^{22,23}$. Both the "experimental" rat and an unfamiliar "partner" rat are placed in the center of the box, and the total duration (seconds) of nonaggressive physical contact (grooming, sniffing, crawling over and under, etc.) initiated by the "experimental" rat is quantified over $5 \mathrm{~min}$. All behaviors were videotaped, and sessions were scored using ANY-maze (Stoelting, Wood Dale, IL) for open field (Stoelting), or by Stephanie D. Fitz (blind to treatments) for social interaction. Each dependent variable for assessment of behavior and radiotelemetry data was respectively analyzed using a one-way ANOVA, or a one-way ANOVA with repeated measures with drug treatment as the main factor and time as repeated measure. In the presence of significant main effects, post hoc tests were conducted using a parametric Fisher's LSD test. Within-subject's time effects were also assessed using Dunnett's one-way analysis with the minute prior to the i.v. infusion used as the control. Statistical significance was accepted with $P<0.05$. All statistical analyses were carried out using SPSS 13.0 (SPSS Inc., IL, USA), and all graphs were generated using SigmaPlot 2001 (SPSS Inc., IL, USA) or Graphpad Prizm 7 Software Inc. for Windows. No randomization was used.

\section{Human studies}

The study protocols and amendments were reviewed and approved by the Medical Ethics Committee of Stichting Beoordeling Ethiek Biomedisch Onderzoek (Assen, The Netherlands). The studies were conducted in compliance with the Declaration of Helsinki and applicable regulatory requirements. All the human studies were conducted at a single center, Centre for Human Drug Research, Leiden, the Netherlands. Written informed consent was obtained from all participants prior to study enrollment. The study design is summarized in Supplementary Fig. S1.

\section{Single-ascending dose (SAD) study of oral JNJ-61393215 in healthy human volunteers}

The SAD study was a phase-1, randomized, doubleblind, placebo-controlled study to assess safety, tolerability, and PK of single increasing oral doses of JNJ-61393215 in healthy adult participants (NCT02812251). 
This was a 3-part study with a total enrollment of 80 participants. The number of participants in each part is the customary sample size employed in such studies. Randomization was used in the assignment of participants to treatment groups using a computer-generated randomization schedule. In Part 1, the single-dose escalation consisted of eight cohorts of healthy male volunteers. In each cohort, six participants received JNJ-61393215 and two participants received matching placebo under fasted conditions. JNJ$61393215(1,2,6,15,30,45,60$, and $90 \mathrm{mg})$ or placebo was administered as a single oral dose. The NeuroCart test battery ${ }^{24}$ was applied to characterize the PD profile of JNJ61393215. CSF levels of JNJ-61393215 and food effect were assessed in Parts 2 and 3, respectively, and those results will be described in separate publications. The study was conducted in a double-blind manner for parts 1 and 3. Blinding was not used in part 3 because all participants received the same dose.

\section{Multiple-ascending dose (MAD) study of oral JNJ- 61393215 in adults}

The MAD study was a randomized, double-blind, placebo-controlled study to investigate safety and tolerability, PK, and PD of JNJ-61393215 in healthy participants under fasted conditions (NCT03007693). Part 1 of the MAD study included four cohorts of male participants (eight participants/cohort, six active, two placebo) with dose levels of $5 \mathrm{mg}$ (Cohort 1), $15 \mathrm{mg}$ (Cohort 2), $45 \mathrm{mg}$ (Cohort 3), and $90 \mathrm{mg}$ (Cohort 4). The number of participants per cohort is the customary sample size employed in early-development dose-escalation studies. Randomization was used in the assignment of participants to treatment groups using a computer-generated randomization schedule. The study was conducted in a doubleblind manner.

\section{$35 \%$ double-inhalation $\mathrm{CO}_{2}$ panic provocation model in humans}

Part 2 (cohort 1) was conducted to establish the anxiolytic PD properties of JNJ-61393215 using the $\mathrm{CO}_{2}$ Tolerance Tester (CTT), a research instrument developed by Maastricht Instruments in collaboration with Maastricht University to induce panic attacks by the protocolized administration of inhaled $35 \% \mathrm{CO}_{2}$. The $\mathrm{CO}_{2}$ challenge procedure was similar to that described in Leibold et al. ${ }^{17}$.

Healthy male participants sensitive to the anxiogenic effects of $35 \% \mathrm{CO}_{2}$ inhalation at screening were randomized to receive JNJ-61393215 (25 mg or $90 \mathrm{mg}$ of QD: extrapolated receptor occupancy at peak concentrations of 93\% and $98.5 \%$, respectively), alprazolam ( $1 \mathrm{mg}$ of bid), or placebo for 7 days. Sensitivity to the $\mathrm{CO}_{2}$ challenge at screening was defined as a change in Panic Symptom List (PSL)-IV score $\geq 4$ with $\geq 1$-point increase for at least four symptoms, and an increase of at least $25 \mathrm{~mm}$ on the Visual Analogue Scale (VAS) for fear-related symptoms ${ }^{17,24}$.

Part 2 of the study applied a four-treatment 3-arm $2 \times 2$ cross-over design, and each participant was randomized to receive either placebo or one of the three active treatments (Supplementary Fig. S1). Assuming that the mean (SD) of paired difference between JNJ groups and placebo observed for this study is $-7(10.3), 12$ participants achieve $84 \%$ power using a paired $t$ test at a onesided alpha of 0.10. A sample size of 36 was used for a four-treatment 3-arm $2 \times 2$ cross-over design.

Participants underwent a $35 \% \mathrm{CO}_{2}$ double-inhalation challenge using the CTT methodology after 6 days of dosing with placebo or active treatment in each cross-over period, and the fear symptoms induced by the $\mathrm{CO}_{2}$ challenge were measured immediately after $\mathrm{CO}_{2}$ inhalation using the panic symptom list IV (PSL-IV). The $\mathrm{CO}_{2}$ challenge was performed $2.5 \mathrm{~h}$ after dosing with JNJ61393215; alprazolam was used as active comparator to establish assay sensitivity as prior studies had shown fearreducing properties of alprazolam in the $\mathrm{CO}_{2}$ challenge after a single dose. Within each arm, the active treatment was compared with matched placebo on PSL-IV total score by using a linear mixed-effect model, controlling for treatment, period, and sequence as a fixed effect, participant as a random effect, and baseline score as a covariate (see supplementary text for eligibility criteria and PK and PD assessments).

\section{Results}

\section{Preclinical studies}

In vitro affinity binding and potency of JNJ-61393215 to OX1R

JNJ-61393215 demonstrated high-affinity binding to the human OX1R and rat OX1R (rOX1R), with negative log of inhibition constant $\left(\mathrm{pK}_{\mathrm{i}}\right)$ values of $8.17 \pm 0.09$ and $8.13 \pm 0.09$, respectively. The binding selectivity of JNJ61393215 for human OX1R compared with human OX2R was substantial ( $\mathrm{pK}_{\mathrm{i}}$ values of 8.17 vs. 6.12). The negative $\log$ of the functional equilibrium dissociation constant $\left(\mathrm{pK}_{\mathrm{B}}\right)$ values for JNJ-61393215 showed good correlation to its $\mathrm{pK}_{\mathrm{i}}$ values for human and $\mathrm{rOX} 1 \mathrm{R}\left(\mathrm{pK} \mathrm{B}_{\mathrm{B}}\right.$ : OX1R, $7.76 \pm$ 0.05 ; rOX1R, $7.72 \pm 0.12$ ). The binding selectivity of JNJ-61393215 at OX1R compared with OX2R was confirmed at the functional level $\left(\mathrm{OX} 1 \mathrm{R} p \mathrm{p}_{\mathrm{B}}=7.76 \mathrm{vs}\right.$. OX2R $\left.\mathrm{pK}_{\mathrm{B}}=6.01\right)$.

JNJ-61393215 was screened in a panel of other receptors, ion channels, transporters, and kinases (Eurofins, France), including adenosine $\left(\mathrm{A}_{1}, \mathrm{~A}_{2 \mathrm{~A}}\right.$, and $\left.\mathrm{A}_{3}\right)$, adrenergic $\left(\alpha_{1}, \alpha_{2}\right.$, and $\left.\alpha_{1}\right)$, angiotensin $\left(\mathrm{AT}_{1}\right)$, dopamine $\left(\mathrm{D}_{1}\right.$ and $\left.\mathrm{D}_{2}\right)$, bradykinin $\left(\mathrm{B}_{2}\right)$, cholecystokinin $\left(\mathrm{CCK}_{\mathrm{A}}\right)$, galanin $\left(\mathrm{GAL}_{2}\right)$, melatonin $\left(\mathrm{ML}_{1}\right)$, muscarinic $\left(\mathrm{M}_{1}, \mathrm{M}_{2}\right.$, and $\left.\mathrm{M}_{3}\right)$, neurotensin $\left(\mathrm{NT}_{1}\right)$, neurokinin $\left(\mathrm{NK}_{2}\right.$ and $\left.\mathrm{NK}_{3}\right)$, opiate $(\mu, \kappa$, and $\delta)$, serotonin $\left(5-\mathrm{HT}_{1 \mathrm{~A}}, 5-\mathrm{HT}_{1 \mathrm{~B}}, 5-\mathrm{HT}_{2 \mathrm{~A}}, 5-\mathrm{HT}_{3}\right.$, 
5- $\mathrm{HT}_{5 \mathrm{~A}}, 5-\mathrm{HT}_{6}$, and $5-\mathrm{HT}_{7}$ ), somatostatin, vasopressin $\left(\mathrm{V}_{1 \mathrm{a}}\right)$, norepinephrine transporter, dopamine transporter, and ion channels (sodium, calcium, potassium, and chloride), and had no significant affinity for any of those binding sites tested $(<50 \%$ inhibition at $10 \mu \mathrm{M})$ other than for OX1R.

\section{Rat brain OX1R occupancy and plasma and brain exposure of JNJ-61393215}

Oral administration of JNJ-61393215 resulted in timeand dose-dependent occupancy of the OX1R in rat tenia tecta, indicating sufficient oral bioavailability and brain penetration (Fig. 1B). Following acute oral administration of $10 \mathrm{mg} / \mathrm{kg}$, maximal OX1R occupancy was observed at $15 \mathrm{~min}(89 \% \pm 6 \%$, Fig. 1B). The level of OX1R occupancy remained above $47 \%$ for the first $8 \mathrm{~h}$, and then dropped below the measurable level of occupancy at $16 \mathrm{~h}$. The brain-to-plasma-concentration ratio ranged from $\sim 0.4$ to 0.7 (Fig. 1C). Overall, the general trend between receptoroccupancy level and plasma exposure was similar (Fig. 1C). The plasma PK was linear, and the plasma concentration associated with $50 \%$ occupancy $\left(\mathrm{EC}_{50}\right)$ was $34 \mathrm{ng} / \mathrm{mL}$ (95\% confidence intervals, $21-56 \mathrm{ng} / \mathrm{mL}$ ).

\section{Effect of JNJ-61393215 on sleep parameters in rats}

Administration of JNJ-61393215 $(10 \mathrm{mg} / \mathrm{kg})$ at the onset of the dark phase showed no alteration in either NREM or REM sleep duration in the 6-h recording period, and there was a significant decrease in the latency of both $\operatorname{NREM}(P<$ $0.01)$ and REM sleep $(P<0.05$; Supplementary Table S1).

\section{JNJ-61393215 selectively promotes REM sleep in OX2R- knockout (KO) mice}

Functional target engagement in vivo was investigated in a model of permanent inhibition of OX2R, and the effects of JNJ-61393215 on sleep-wake states were examined in $\mathrm{KO}$ mice lacking the $\mathrm{OX} 2 \mathrm{R}$ relative to their corresponding WT mice. In OX2R KO mice, oral dosing of JNJ-61393215 $(30 \mathrm{mg} / \mathrm{kg})$ compared with vehicle at $2 \mathrm{~h}$ into the light phase significantly reduced the latency for REM sleep $(P<0.01)$ and prolonged the time spent in REM sleep during the first $2 \mathrm{~h}$ post treatment $(P<0.05$; Supplementary Fig. S2A), but did not alter REM sleep in WT mice $(P>0.05$; Supplementary Fig. 2B). As opposed to REM sleep parameters, NREM sleep latency and duration were not significantly affected by the JNJ61393215 in either genotype $(P>0.05$; Supplementary Figs. S2C and S2D).

\section{Effect of JNJ-61393215 on $\mathrm{CO}_{2}$-induced fear and cardiovas- cular response in rats}

JNJ-61393215 was tested in a rat model of $20 \% \mathrm{CO}_{2}-$ induced panic, using oral doses of 3,10 , and $30 \mathrm{mg} / \mathrm{kg}$ administered 30-50 min prior to the $\mathrm{CO}_{2}$ challenge (when a baseline was established for physiological measures, see Fig. 2). The less active enantiomer of JNJ-61393215, JNJ63821238 , was included as a negative control.

Repeated measure ANOVA with treatment as the main factor and time as the repeated measure revealed the following:

- A one-way ANOVA revealed differences between groups $\mathrm{F}(4,51)=5.2, P<0.001$ (Fig. 2A). Ficher's LSD post hoc test revealed that the $\mathrm{CO}_{2}$-induced reduction in social interaction behaviors (indicating fear state) was blocked in rats pretreated with the 10 or $30 \mathrm{mg} / \mathrm{kg}$ dose of JNJ-61393215.

- A significant increase in mean arterial blood pressure over time $\mathrm{F}(14,518)=83.1, P<0.001$, but no treatment $\times$ time interaction was observed $\mathrm{F}$ $(42,518)=0.7, \quad P=0.918$. There were also no significant differences in mean arterial blood pressure noted at baseline between treatment groups $\mathrm{F}(3,40)=0.5, P=0.708$ (Fig. 2B).

- A significant decrease in heart rate over time $F$ $(14,518)=53.6, P<0.001$, and a treatment $\times$ time interaction was observed $\mathrm{F}(42,518)=2.1, P<0.001$, and a Fisher's least-square difference (LSD) post hoc test protected by an ANOVA at each time point revealed that JNJ-61393215 attenuated $\mathrm{CO}_{2}$-induced bradycardia responses at two time points (8 and $9 \mathrm{~min})$ post $\mathrm{CO}_{2}(P<0.05$, Fig. $2 \mathrm{C})$. Also, no differences in heart rate were detected at baseline between treatment groups $\mathrm{F}(3,40)=0.5, P=0.667$.

- A significant increase was detected in general motor activity over time $\mathrm{F}(14,518)=10.7, P<0.001$, but no treatment $\times$ time interaction was observed $\mathrm{F}(42,518)$ $=0.9, P=0.658$. No differences in general motor activity were detected at baseline between treatment groups $\mathrm{F}(3,40)=0.4, P=0.757$ (Fig. 2D).

- A significant decrease was detected in core body temperature over time $\mathrm{F}(14,518)=146.3, P<0.001$, but no treatment $\times$ time interaction was observed $\mathrm{F}(42,518)=1.2, P=0.202$. Also, no differences in core body temperature were detected at baseline between treatment groups $\mathrm{F}(3,40)=0.2, P=0.912$ (Fig. 2E).

Mean total and unbound JNJ-61393215 plasma levels measured at $30 \mathrm{~min}$ at $10 \mathrm{mg} / \mathrm{kg}$ were $535 \mathrm{ng} / \mathrm{mL}$ and $86 \mathrm{ng} / \mathrm{mL}$, respectively, while at $30 \mathrm{mg} / \mathrm{kg}$, mean total and unbound levels were $2698 \mathrm{ng} / \mathrm{mL}$ and $432 \mathrm{ng} / \mathrm{mL}$, respectively (Supplementary Table S2).

\section{Clinical studies \\ Pharmacokinetic profiles and safety of single-ascending dose (SAD) of JNJ-61393215 in humans (part 1)}

In the SAD study, 72 male participants (aged 18-52 years, mean [SD] age: 31 [15.06] years; BMI: $23.1 \mathrm{~kg} / \mathrm{m}^{2}$, median 22.7; range 19-30) were included. Females were 


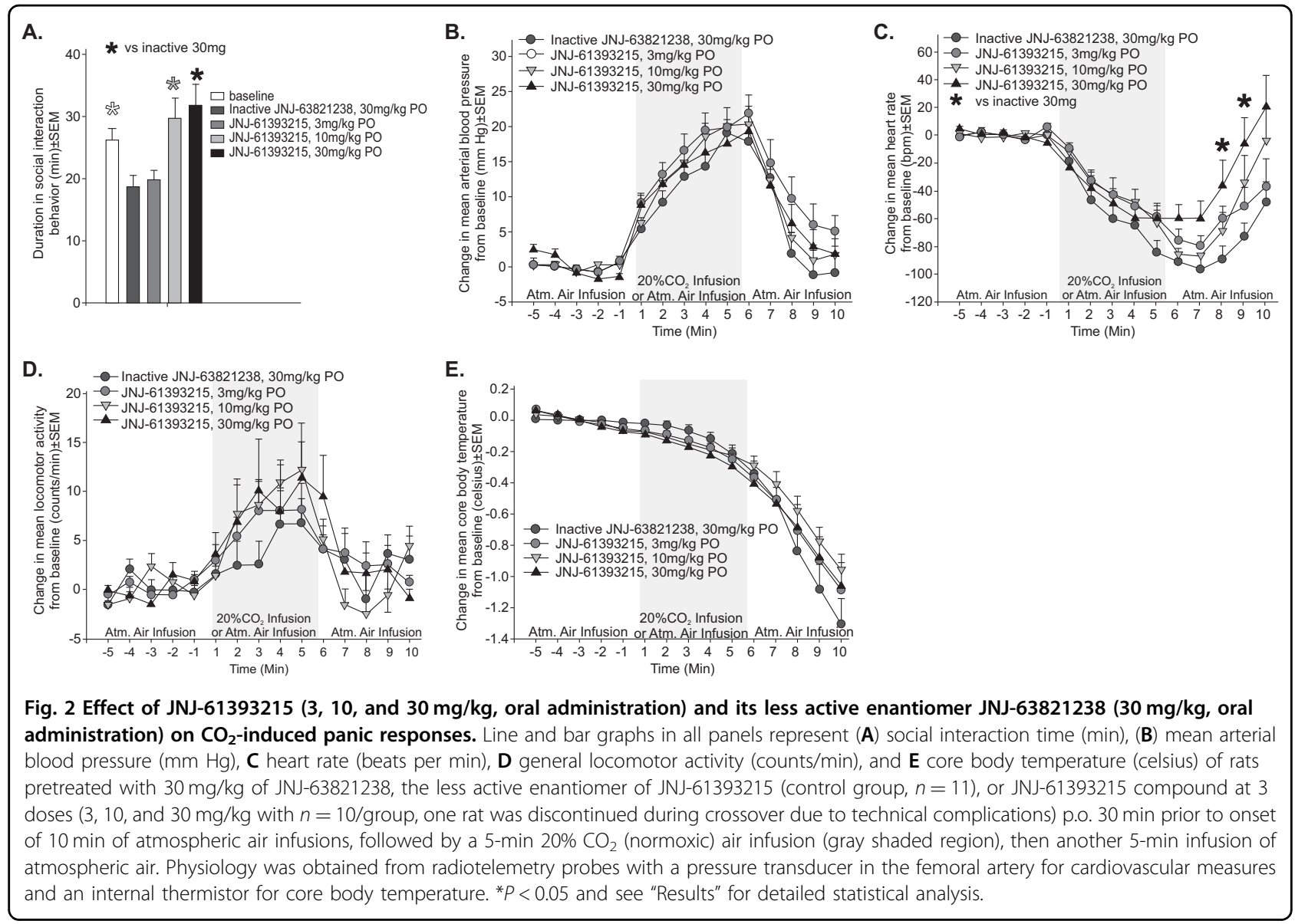

excluded due to lack of preclinical reproductive and developmental toxicity results available at the time the study was conducted. The study consisted of 8 cohorts; 6 male participants per cohort received a single dose of JNJ61393215 , ranging from 1 to $90 \mathrm{mg}(n=48)$. The majority $(87.5 \%)$ of participants in the JNJ-61393215 treatment groups were Caucasians. The mean values of $C_{\max }$, $\mathrm{AUC}_{\text {last}}$, and $\mathrm{AUC}_{\infty}$ of JNJ-61393215 increased doseproportionally with increasing dose up to $30 \mathrm{mg}$ (Supplementary Table S3).

The most frequently reported treatment-emergent adverse events (TEAEs) in participants dosed with JNJ$61393215(1-90 \mathrm{mg})$ were somnolence $(n=8,16.7 \%)$ and headache $(n=23,47.9 \%)$. In the placebo group, six $(33 \%)$ participants experienced headache, and three (16.7\%) participants reported somnolence (Supplementary Table S4).

\section{Pharmacokinetic profiles and safety of multiple-ascending dose (MAD) of JNJ-61393215 in humans (parts 1 and 2)}

Overall, 32 male participants (mean [SD] age: 30.5 [11.2] years) in Part 1 and 39 male participants (mean [SD] age: 28.2 [8.0] years) in Part 2 were included in the MAD study. In the JNJ-61393215 treatment groups, the majority $(\sim 80 \%)$ of participants were Caucasians with mean BMI of 22.9 (2.8) kg/m² (range 17.9-29.4). On days 1 and 7, mean $C_{\max }$ and $\mathrm{AUC}_{24 \mathrm{~h}}$ increased with increasing doses (Fig. 3; Supplementary Table S5). Overall, the mean values for the dose-normalized PK parameters on days 1 and 7 slightly decreased with increasing doses, suggesting a less-than dose-proportional increase over the dose range of 5-90 mg of JNJ-61393215 (Supplementary Table S5).

For Part 1, the most common TEAEs (reported by $\geq 10 \%$ of participants) in all JNJ-61393215 treatment groups were headache $(33.3 \%)$, somnolence $(29.2 \%)$, nausea (16.7\%), nasopharyngitis (16.7\%), dysgeusia (12.5\%), and epistaxis (8.3\%; Supplementary Table S4). In the placebo group, three participants (37.5\%) experienced headache and three participants experienced somnolence (37.5\%) and two (25\%) participants experienced dysgeusia (Supplementary Table S4). None of the TEAEs were considered severe. There were no consistent treatmentrelated effects in clinical laboratory parameters, and no abnormalities were observed during physical examination.

\section{Human pharmacodynamic results (MAD study part 2)}

Treatment with JNJ-61393215 (90 mg) significantly reduced fear symptoms induced by double inhalation of $35 \% \mathrm{CO}_{2}$ in healthy volunteers according to the primary 


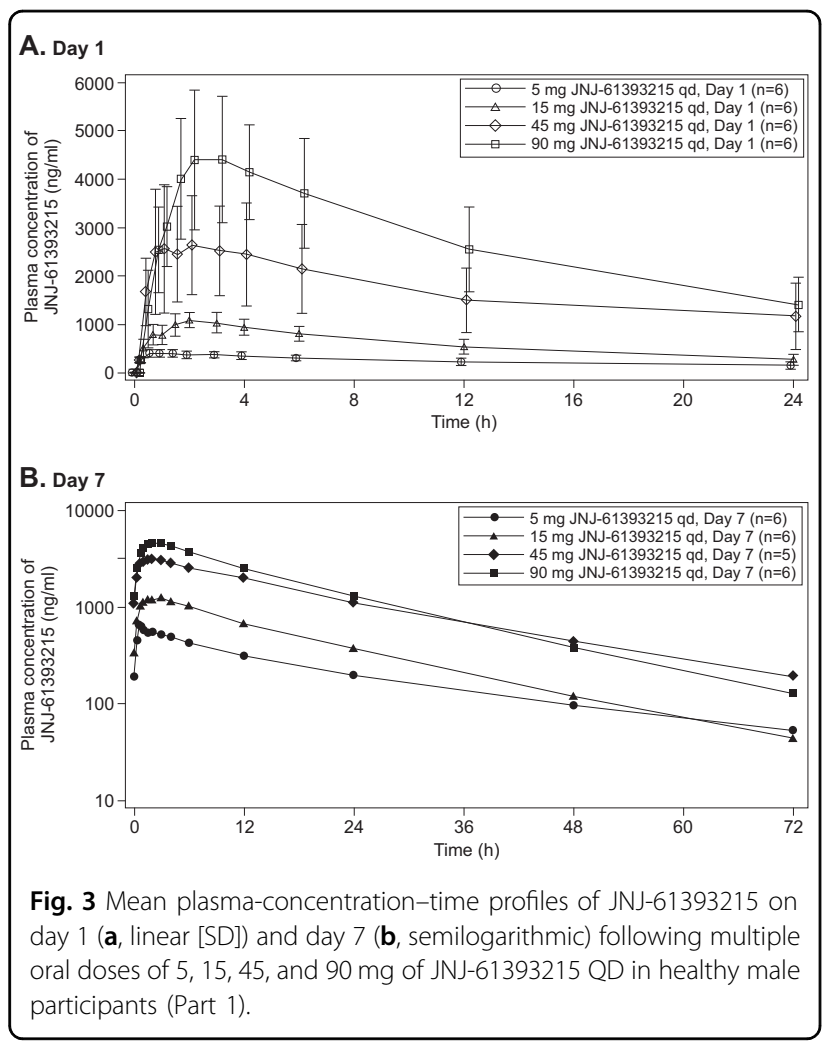

outcome measure, namely the change PSL-IV total score (difference of least-square [LS] means (SE): $-2.3(0.9), P<$ 0.02 ); a significant anxiolytic effect was also demonstrated for a therapeutic dose of active comparator alprazolam (difference of LS means (SE): $-3.4 \quad(1.5), \quad P<0.03$ ), demonstrating assay sensitivity. Individual item changes after active treatment or placebo are presented in Table 1 in a descriptive manner, to highlight the clinical implication of the PSL-IV total score analysis. Statistical analyses were not performed on individual item change, as the study was not powered to look at individual item changes after applying appropriate corrections for multiple comparisons given the relatively small sample size (which was based on power calculations using the estimated PSL-IV total score change only). The anxiolytic effect of JNJ-61393215 was observed in most participants, and was driven by a reduction in severity of $9 / 13$ items of the PSL-IV, suggesting a broad anxiolytic effect (Table 1). The low dose of JNJ-61393215 (25 mg) was associated with a nonsignificant decrease in fear symptoms (Fig. 4). Mean unbound $C_{\max }$ $90 \mathrm{mg}(68 \mathrm{ng} / \mathrm{mL})$ on day 7 was comparable to the mean unbound $\mathrm{C}_{\max }$ obtained in the $\mathrm{CO}_{2}$-induced reduction in social interaction behavior paradigm at $10 \mathrm{mg} / \mathrm{kg}(86 \mathrm{ng} /$ $\mathrm{mL}$ ) in rats (Supplementary Table S2).

\section{Cardiovascular parameters}

JNJ-61393215 and alprazolam did not have any significant effects on cardiovascular parameters, such as blood pressure or heart rate (data not shown). Considerable variability of physiological parameters at baseline and after the $\mathrm{CO}_{2}$ challenge was observed across the study population, which is consistent with the results of a recent meta-analysis ${ }^{25}$.

\section{Discussion}

In the preclinical studies described in this report, we characterized the novel selective OX1R antagonist, JNJ61393215, as a clinical candidate for the treatment of panic and anxiety disorders. We also demonstrated that pretreatment of rats with JNJ-61393215 attenuates the anxiety- and panic-like responses elicited by $20 \% \mathrm{CO}_{2}$ inhalation. Safety, PK, and PD properties of JNJ-61393215 in first-in-human single- and multiple-ascending dose studies were described, and an anxiolytic effect of JNJ61393215 was demonstrated in healthy participants using a modified $\mathrm{CO}_{2}$ challenge assay to induce panic symptoms. This is the first report of anxiolytic effects of a selective OX1R antagonist in humans.

In the in vitro studies, JNJ-61393215 demonstrated high affinity, potency, and selectivity for OX1R with an approximate $2-\log$ selectivity ratio relative to OX2R. The in vivo occupancy of OX1R as assessed by ex vivo receptor analysis showed time- and dose-dependent OX1R occupancy in rat brain. In the rat study, JNJ-61393215 showed no effect on spontaneous sleep; however, a decrease in latency to NREM and REM was observed and was similar to previously observed reports with a different OX1R antagonist JNJ-54717793 ${ }^{11}$. Our preclinical findings corroborate the results of other studies wherein selective OX1R antagonists, in contrast to selective OX2R antagonists, minimally affect sleep-wake states in baseline conditions $^{21,26,27}$. EEG was obtained during the rat's dark/ active phase that also corresponds to the daytime/active phase in humans when the drug was given. The dark phase in rodents is the optimal phase to detect any hypnotic activity for orexin-receptor antagonists (due to higher orexin levels) and the only circadian phase where all orexin $\mathrm{KO}$ (peptide and receptors) show the sleep phenotype. We also tested JNJ-61393215 in WT mice at a higher dose during the light phase, in which it proved to be devoid of sleep-promoting effects. Consistent with our previous results on simultaneous pharmacological OX1R and OX2R blockade, JNJ-61393215 promoted REM sleep in OX2R KO mice, but not in WT mice, demonstrating that the compound engaged OX1Rs and elicited a specific OX1R blockade.

While JNJ-61393215 showed no effect on baseline cardiovascular, temperature, or locomotor activity, it blocked $\mathrm{CO}_{2}$-induced fear in the social interaction test at $10 \mathrm{mg} /$ $\mathrm{kg}$ and $30 \mathrm{mg} / \mathrm{kg}$. In addition, an attenuation of the bradycardia response to $\mathrm{CO}_{2}$ challenge was also observed at $30 \mathrm{mg} / \mathrm{kg}$ dosing. 
Table 1 PSL-IV individual item change scores post $\mathrm{CO}_{2}$ challenge.

\begin{tabular}{|c|c|c|c|c|c|c|}
\hline & Placebo & $\begin{array}{l}\text { JNJ-61393215 } \\
25 \mathrm{mg} \text { of QD }\end{array}$ & Placebo & $\begin{array}{l}\text { JNJ-61393215 } \\
90 \mathrm{mg} \text { of QD }\end{array}$ & Placebo & $\begin{array}{l}\text { Alprazolam } \\
1 \mathrm{mg} \text { of BID }\end{array}$ \\
\hline Dizziness & $1.9(1.08)$ & $1.3(1.22)$ & $2.3(1.06)$ & $1.8(1.09)$ & $2.1(0.92)$ & $1.7(1.19)$ \\
\hline Choking/gasping for breath & $1.8(0.83)$ & $1.9(0.90)$ & $2.5(1.38)$ & $1.8(1.28)$ & $2.1(0.92)$ & $1.9(1.30)$ \\
\hline Hot flashes/cold shivers & $0.3(0.75)$ & $0.3(0.65)$ & $0.9(1.56)$ & $0.5(1.33)$ & $0.8(1.25)$ & $0.2(0.40)$ \\
\hline Nausea & $0.4(0.51)$ & $0.2(0.39)$ & $0.7(1.23)$ & $0.5(1.33)$ & $0.3(0.61)$ & $0.1(0.30)$ \\
\hline Palpitations & $1.9(1.08)$ & $1.3(1.15)$ & $1.8(1.27)$ & $1.8(1.28)$ & $1.9(0.95)$ & $1.3(0.90)$ \\
\hline Sweating & $0.7(0.49)$ & $0.8(0.94)$ & $1.3(1.29)$ & $0.9(0.86)$ & $1.3(1.14)$ & $1.0(0.89)$ \\
\hline Shortness of breath & $1.6(1.00)$ & $1.8(0.97)$ & $2.3(1.15)$ & $2.1(1.32)$ & $2.2(0.89)$ & $1.9(1.22)$ \\
\hline Numb/tingling & $0.8(0.94)$ & $0.7(1.07)$ & $1.5(1.0)$ & $1.2(1.36)$ & $0.9(0.95)$ & $0.7(0.79)$ \\
\hline $\begin{array}{l}\text { Depersonalization/ } \\
\text { derealization }\end{array}$ & $0.5(0.80)$ & $0.6(0.79)$ & $1.2(1.47)$ & $1.2(1.41)$ & $1.1(1.10)$ & $0.8(0.98)$ \\
\hline Fear of dying & $0.1(0.29)$ & $0.1(0.29)$ & $0.3(0.89)$ & $0.4(1.12)$ & $0.3(0.73)$ & $0.1(0.30)$ \\
\hline Fear of losing control & $0.3(0.65)$ & $0.3(0.65)$ & $0.4(0.90)$ & $0.4(0.87)$ & $0.3(0.61)$ & $0.4(0.50)$ \\
\hline Chest pain discomfort & $0.3(0.45)$ & $0.2(0.39)$ & $0.8(1.14)$ & $0.7(1.18)$ & $0.1(0.36)$ & $0(0)$ \\
\hline Trembling/shaking & $1.3(0.87)$ & $1.3(0.97)$ & $1.6(1.51)$ & $1.4(1.39)$ & $1.1(1.27)$ & $1.2(1.54)$ \\
\hline
\end{tabular}

BID twice daily, PSL-IV panic symptom list IV, SD standard deviation, $Q D$ once daily. Data are represented as mean (SD).

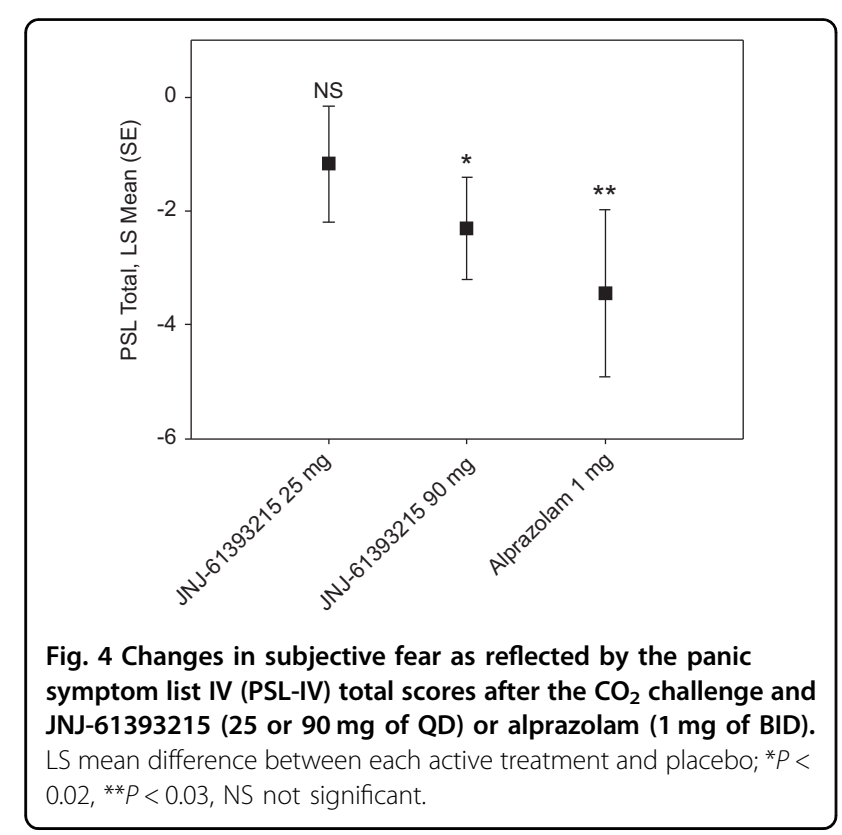

The efficacy observed in the rat $\mathrm{CO}_{2}$ challenge is unlikely to be driven by nonspecific effects, as demonstrated by the lack of effect on baseline locomotor or autonomic activity, and by the lack of effect of the less active enantiomer JNJ-63821238. A high level of target engagement $(>90 \%)$ was required to demonstrate efficacy in the $\mathrm{CO}_{2}$ challenge, as previously observed with other OX1R antagonists (SB-334867, Compound 56 and JNJ$54717793)^{11,12,28}$. These findings are consistent with the hypothesized role of OX1R signaling in the mechanism underlying $\mathrm{CO}_{2}$ challenge-induced fear, as exposing rats to higher concentration of $\mathrm{CO}_{2}$ depolarizes orexin neurons by interacting with $\mathrm{pH} / \mathrm{CO}_{2}$-chemosensitive $\mathrm{K}^{+}$ channels $^{29}$. This results in subsequent release of orexin at postsynaptic sites in brain and brainstem regions to mobilize anxiety-like behavior, hypertension, and increased ventilatory responses ${ }^{28,30}$.

Notably, the unbound mean JNJ-61393215 plasma concentrations at which $\mathrm{CO}_{2}$-induced fear in rats was inhibited $(86-432 \mathrm{ng} / \mathrm{mL})$, were comparable to those associated with the reduction of experimental $35 \% \mathrm{CO}_{2}$ induced fear in humans $(68 \mathrm{ng} / \mathrm{mL})$. In fact, the unbound plasma exposure in rats predicted the exposure at which inhibition of $\mathrm{CO}_{2}$-induced fear could be expected in humans.

In humans, JNJ-61393215 demonstrated an acceptable safety profile for both after single-dose and multiple-dose administration up to $90 \mathrm{mg}$, as suggested by the findings in the SAD and the MAD studies in humans. The generalizability of these results is limited; however, since the SAD and MAD studies did not include women, the preclinical reproductive and developmental toxicology experiments had not been completed when those studies were executed. It is important to note that the maximal doses administered in the SAD and the MAD studies, which correspond to extrapolated receptor occupancy $>95 \%$, were not associated with somnolence, providing evidence in humans that the soporific properties of dual orexin-receptor antagonists are associated with orexin-2 receptors. The majority of TEAEs were mild in severity, and their frequency was comparable to placebo. After administration of a single dose of JNJ-61393215, peak and total exposure increased in a dose-proportional manner up to $30 \mathrm{mg}$. JNJ-61393215 did not produce any pharmacodynamic effects as a single dose up to $90 \mathrm{mg}$ in healthy male subjects, consistent with preclinical data that 
show no detectable pharmacodynamic or behavioral effects in naive/unchallenged animals.

The safety profile of JNJ-61393215 is consistent with the recently reported clinical data for another selective OX1R antagonist, ACT-539313, where the most commonly reported adverse events were somnolence and headache ${ }^{31}$.

JNJ-61393215 also showed anxiolytic properties in a human experimental model of panic induced by inhalation of high concentrations of $\mathrm{CO}_{2}$. Several aspects related to the $\mathrm{CO}_{2}$ challenge merit comment, especially in light of the methodological heterogeneity of prior studies that implemented similar paradigms. In the human MAD study, we opted for double-breath inhalation of $35 \% \mathrm{CO}_{2}$, which is considered an experimental model of panic ${ }^{17}$, in contrast to other $\mathrm{CO}_{2}$-challenge studies that used $5 \%$ or $7 \% \mathrm{CO}_{2}$ inhaled over prolonged periods, and has been proposed as a model for generalized anxiety disorder ${ }^{32}$. $\mathrm{CO}_{2}$ is hypothesized to acutely disturb the brain acid-base homeostasis in both medullary respiratory neurons and serotonergic midbrain raphe neurons.

The choice of a challenge model more relevant for panic symptoms to test PD effects of JNJ-6139215 is consistent with the mechanism of action of OXR1 inhibition as well as prior preclinical studies ${ }^{21}$. The $35 \%$ double-inhalation $\mathrm{CO}_{2}$ challenge has been extensively validated with several anxiolytic treatments, such as acute dosing of benzodiazepines, chronic dosing with selective serotonin-reuptake inhibitors, and chronic dosing with tricyclic antidepressants ${ }^{17,19,20}$. The panicolytic effects of JNJ61393215 and alprazolam were also tested at steady state and not after a single dose, while previous studies with benzodiazepines have tested their panicolytic effects in the $\mathrm{CO}_{2}$ challenge only after a single dose. Together, this study provided further validation for the $\mathrm{CO}_{2}$ challenge as a test sensitive to the effects of alprazolam even after multiple doses, supporting the translational value of this challenge in anxiolytic drug development.

The incomplete cross-over design was an additional strength to the conduct of $\mathrm{CO}_{2}$-challenge experiments, and allowed testing of each experimental treatment versus placebo in a relatively small number of participants. Also, we limited enrollment to participants who were sensitive to $\mathrm{CO}_{2}$ inhalation at screening in the MAD study. Previous studies have shown that healthy volunteers manifest lower sensitivity to the panic-like effects of $\mathrm{CO}_{2}$ inhalation than patients with panic disorder ${ }^{33}$; therefore, the implementation of an enrichment strategy was deemed necessary to avoid potential floor effects, which may have compromised the detection of potential PD anxiolytic effects induced by administration of JNJ-61393215 and alprazolam. JNJ-61393215 at $90 \mathrm{mg}$ showed a robust anxiolytic effect, driven by a decrease in most items on the PSL-IV, similar to the active comparator, alprazolam, supporting the potential anxiolytic effect properties of
OX1R antagonists. The reduction in anxiety symptoms observed after administration of JNJ-61393215 $90 \mathrm{mg}$ and alprazolam was not paralleled by any changes in cardiovascular parameters: we observed marked interindividual variability in the changes in blood pressure and heart rate elicited by $\mathrm{CO}_{2}$ inhalation, as well as in the effects of JNJ61393215 and alprazolam on those measures, a finding consistent with a recent meta-analysis that highlighted the large variability and limited interpretability of the effects of the $\mathrm{CO}_{2}$ challenge on cardiovascular parameters ${ }^{25}$.

Notably, the extant preclinical data suggest that OX1R antagonists may hold anxiolytic effects that extend beyond panic anxiety. For example, in rodents, the autonomic and behavioral responses to stress were attenuated by pretreatment with OX1R antagonists ${ }^{8}$.

In conclusion, we demonstrated for the first time in humans the panicolytic effects of a selective OX1R antagonist, JNJ-61393215, with an acceptable safety profile. In addition, this study supported the validity of the $\mathrm{CO}_{2}$-exposure challenge as a translational, cross-species experimental model for panic. These results collectively support further evaluation of JNJ-61393215 efficacy in clinical studies as a potential treatment for patients with panic or other anxiety disorders, and in mood disorders associated with anxious hyperarousal.

\section{Acknowledgements}

We acknowledge Michelle Wennerholm, Tatiana Koudriakova, Anthony Ndifor, and Kevin Sharp for their contributions to the studies. Lakshmi Kasthurirangan, PhD and Shweta Pitre, CMPP (both SIRO Clinpharm Pvt. Ltd.) provided medical writing assistance, and Ellen Baum, PhD (Janssen Global Services, LLC) provided additional editorial support for this paper. We thank Stephanie Fitz (Indiana University, School of Medicine, Indianapolis) for technical support on animal model experiments and data generation. Giacomo Salvadore, Pascal Bonaventure, Brian Lord, Brock Shireman, Terry Lebold, Diane Nepomuceno, Christine Dugovic, Cathy Bleys, Kanaka Tatikola, Bart Remmerie, John Moyer, Abigail Nash, Luc GM Van Nueten, and Wayne C Drevets are employees at Janssen Research \& Development, LLC. Gabriel E Jacobs, Sander Brooks, and Rob Zuiker are employees at the Centre for Human Drug Research, Leiden, the Netherlands. Also supported by NIA 1K01AG044466-01A1 to P.L.J., and NIMH R01 MH52619 and R01 MH65702 to A.S. This study is supported by Janssen Research \& Development, LLC, USA. Janssen Research \& Development, LLC facilitated the study design, provided writing assistance and editorial support for the paper, and reviewed and approved the paper prior to submission. The authors independently collected the data, interpreted the results, and had the final decision to submit the paper for publication.

\section{Author details \\ ${ }^{1}$ Janssen Research \& Development, LLC, Titusville, NJ, USA. ${ }^{2}$ Janssen Research \& Development, LLC, San Diego, CA, USA. ${ }^{3}$ Departments of Psychiatry, and Pharmacology, Indiana University, School of Medicine, Indianapolis, IN, USA. ${ }^{4}$ Department of Anatomy, Physiology and Cell Biology, Indiana University, School of Medicine, Indianapolis, IN, USA. ${ }^{5}$ Centre for Human Drug Research, Leiden, The Netherlands. ${ }^{6}$ Leiden University Medical Center, Leiden, The Netherlands. ${ }^{7}$ Janssen Research \& Development, LLC, Beerse, Belgium. ${ }^{8}$ Janssen Scientific Affairs, LLC, Titusville, NJ, USA. ${ }^{9}$ Department of Psychiatry, Leiden University Medical Center, Leiden, The Netherlands. ${ }^{10}$ Research School for Mental Health and Neuroscience, Maastricht University, Maastricht, The Netherlands}

Conflict of interest

G.S., P.B., B.L., B.T.S., T.P.L., D.N., C.D., C.B., K.T., B.R., J.M., A.N., L.G.M.V.N., and W.C. D. are current or former employees of Janssen Research \& Development, LLC 
and own stock/stock options in Johnson \& Johnson. A.S. has received research awards from Eli Lilly and Co., Johnson \& Johnson, and AstraZeneca. R.Z., S.B., and G.E.J. were the study investigators and employees of CHDR; P.J. and K.S. do not have any conflict of interests to declare. All authors participated in the original design of the studies, supervising recruitment and monitoring of data quality, and contributed to the data interpretation, development, and review of this paper, and confirm that they have read the journal's position on issues involved in ethical publication, and affirm that this report is consistent with those guidelines. All authors met ICMJE criteria, and all those who fulfilled those criteria are listed as authors. All authors had access to the study data, provided direction, and comments on the paper, made the final decision about where to publish these data, and approved submission to this journal.

\section{Publisher's note}

Springer Nature remains neutral with regard to jurisdictional claims in published maps and institutional affiliations.

Supplementary Information accompanies this paper at (https://doi.org/ 10.1038/s41398-020-00937-9).

Received: 30 April 2020 Revised: 8 July 2020 Accepted: 14 July 2020 Published online: 07 September 2020

\section{References}

1. Peyron, $\mathrm{C}$. et al. Neurons containing hypocretin (orexin) project to multiple neuronal systems. J. Neurosci. 18, 9996-10015 (1998).

2. de Lecea, L. et al. The hypocretins: hypothalamus-specific peptides with neuroexcitatory activity. Proc. Natl Acad. Sci. USA 95, 322-327 (1998).

3. James, M. H., Campbell, E. J. \& Dayas, C. V. Role of the orexin/hypocretin system in stress-related psychiatric disorders. Curr. Top. Behav. Neurosci. 33 197-219 (2017).

4. Brisbare-Roch, C. et al. Promotion of sleep by targeting the orexin system in rats, dogs and humans. Nat. Med. 13, 150-155 (2007).

5. Mang, G. M. et al. The dual orexin receptor antagonist almorexant induces sleep and decreases orexin-induced locomotion by blocking orexin 2 receptors. Sleep 35, 1625-1635 (2012).

6. Bonaventure, $\mathrm{P}$. et al. Characterization of JNJ-42847922, a selective orexin-2 receptor antagonist, as a clinical candidate for the treatment of insomnia. J. Pharm. Exp. Ther. 354, 471-482 (2015).

7. Gozzi, A., Lepore, S., Vicentini, E., Merlo-Pich, E. \& Bifone, A. Differential effect of orexin-1 and CRF-1 antagonism on stress circuits: a fMRI study in the rat with the pharmacological stressor Yohimbine. Neuropsychopharmacology $\mathbf{3 8}$, 2120-2130 (2013).

8. Sears, R. M. et al. Orexin/hypocretin system modulates amygdala-dependent threat learning through the locus coeruleus. Proc. Natl Acad. Sci. USA 110, 20260-20265 (2013).

9. Flores, A., Saravia, R., Maldonado, R. \& Berrendero, F. Orexins and fear: implications for the treatment of anxiety disorders. Trends Neurosci. 38, 550-559 (2015).

10. Flores, A. et al. The hypocretin/orexin system mediates the extinction of fear memories. Neuropsychopharmacology 39, 2732-2741 (2014)

11. Bonaventure, P. et al. Evaluation of JNJ-54717793 a novel brain penetrant selective orexin 1 receptor antagonist in two rat models of panic attack provocation. Front. Pharm. 8, 357 (2017).
12. Bonaventure, P. et al. A selective orexin-1 receptor antagonist attenuates stress-induced hyperarousal without hypnotic effects. J. Pharm. Exp. Ther. 352, 590-601 (2015).

13. Johnson, P. L. et al. A key role for orexin in panic anxiety. Nat. Med. 16, 111-115 (2010)

14. Nishino, S., Ripley, B., Overeem, S., Lammers, G. J. \& Mignot, E. Hypocretin (orexin) deficiency in human narcolepsy. Lancet 355, 39-40 (2000).

15. Johnson, P. L., Molosh, A., Fitz, S. D., Truitt, W. A. \& Shekhar, A. Orexin, stress, and anxiety/panic states. Prog. Brain Res. 198, 133-161 (2012).

16. Johnson, P. L. et al. Activation of the orexin 1 receptor is a critical component of $\mathrm{CO}_{2}$-mediated anxiety and hypertension but not bradycardia. Neuropsychopharmacology 37, 1911-1922 (2012).

17. Leibold, N. K. et al. CO2 exposure as translational cross-species experimental model for panic. Transl. Psychiatry 6, e885 (2016).

18. Dugovic, C. et al. Orexin-1 receptor blockade dysregulates REM sleep in the presence of orexin-2 receptor antagonism. Front. Neurosci. 8, 28 (2014).

19. Sanderson, W. C., Wetzler, S. \& Asnis, G. M. Alprazolam blockade of $\mathrm{CO}_{2}-$ provoked panic in patients with panic disorder. Am. J. Psychiatry 151, 1220-1222 (1994)

20. Nardi, A. E. et al. Clonazepam for the treatment of panic disorder. Curr. Drug Targets 14, 353-364 (2013).

21. Dugovic, C. et al. Blockade of orexin-1 receptors attenuates orexin-2 receptor antagonism-induced sleep promotion in the rat. J. Pharm. Exp. Ther. 330, 142-151 (2009).

22. Johnson, P. L., Hollis, J. H., Moratalla, R., Lightman, S. L. \& Lowry, C. A. Acute hypercarbic gas exposure reveals functionally distinct subpopulations of serotonergic neurons in rats. J. Psychopharmacol. 19, 327-341 (2005).

23. Sanders, S. K. \& Shekhar, A. Regulation of anxiety by GABAA receptors in the rat amygdala. Pharm. Biochem. Behav. 52, 701-706 (1995).

24. Groeneveld, G. J., Hay, J. L. \& Van Gerven, J. M. Measuring blood-brain barrier penetration using the NeuroCart, a CNS test battery. Drug Discov. Today Technol. 20, 27-34 (2016).

25. Liu, J. J. W., Ein, N., Gervasio, J. \& Vickers, K. Subjective and physiological responses to the $35 \%$ carbon dioxide challenge in healthy and non-clinical control populations: a meta-analysis and systematic review. Anxiety Stress Coping 32, 216-230 (2019).

26. Smith, M. I., Piper, D. C., Duxon, M. S. \& Upton, N. Evidence implicating a role for orexin-1 receptor modulation of paradoxical sleep in the rat. Neurosci. Lett. 341, 256-258 (2003).

27. Gozzi, A. et al. Functional magnetic resonance imaging reveals different neural substrates for the effects of orexin-1 and orexin-2 receptor antagonists. PLoS ONE 6, e16406 (2011).

28. Johnson, P. L. et al. Orexin 1 and 2 receptor involvement in $\mathrm{CO}_{2}$-induced panic-associated behavior and autonomic responses. Depress Anxiety 32, 671-683 (2015).

29. Gonzalez, J. et al. Deletion of TASK1 and TASK3 channels disrupts intrinsic excitability but does not abolish glucose or $\mathrm{pH}$ responses of orexin/hypocretin neurons. Eur. J. Neurosci. 30, 57-64 (2009).

30. Johnson, P. L. et al. Orexin 1 receptors are a novel target to modulate panic responses and the panic brain network. Physiol. Behav. 107, 733-742 (2012).

31. Kaufmann, P., Ort, M., Golor, G., Kornberger, R. \& Dingemanse, J. First-in-human study with ACT-539313, a novel selective orexin-1 receptor antagonist. Br. J. Clin. Pharmacol. 86, 1377-1386 (2020).

32. Bailey, J. E., Kendrick, A., Diaper, A., Potokar, J. P. \& Nutt, D. J. A validation of the $7.5 \% \mathrm{CO}_{2}$ model of $\mathrm{GAD}$ using paroxetine and lorazepam in healthy volunteers. J. Psychopharmacol. 21, 42-49 (2007).

33. Kent, J. M. et al. Specificity of panic response to $\mathrm{CO}_{2}$ inhalation in panic disorder: a comparison with major depression and premenstrual dysphoric disorder. Am. J. Psychiatry 158, 58-67 (2001). 